\title{
SERIES INTRODUCTION
}

\section{Do tumor-suppressive mechanisms contribute to organism aging by inducing stem cell senescence?}

\author{
Pier Giuseppe Pelicci \\ Department of Experimental Oncology, European Institute of Oncology and FIRC Institute of Molecular Oncology, \\ Milan, Italy
}

\begin{abstract}
Stem/progenitor cells ensure tissue and organism homeostasis and might represent a frequent target of transformation. Although these cells are potentially immortal, their life span is restrained by signaling pathways ( $\mathrm{p} 19-\mathrm{p} 53$; $\mathrm{p} 16-\mathrm{Rb})$ that are activated by DNA damage (telomere dysfunction, environmental stresses) and lead to senescence or apoptosis. Execution of these checkpoint programs might lead to stem cell depletion and organism aging, while their inactivation contributes to tumor formation.
\end{abstract}

J. Clin. Invest. 113:4-7 (2004). doi:10.1172/JCI200420750.

Telomeres form high-order chromatin structures that cap the ends of eukaryotic chromosomes. They contain thousands of double-stranded repeats (TTAGGG) and terminate with a single-stranded 3 '-extension, which, together with specific telomeric proteins, participates in the formation of the terminal loop structure (telomere cap). Telomeres are synthesized by telomerase, a cellular reverse transcriptase that adds TTAGGG repeats onto preexisting telomeres. In cells that do not express telomerase, TTAGGG repeats are lost at each cell division, and, when telomeres reach a critical length, a checkpoint is triggered that drives cells into a metabolically active state of irreversible growth arrest, termed replicative senescence. Therefore, telomere shortening has generally been regarded as a counting mechanism that limits the mitotic potential of any cell type. In this view, cellular senescence can be considered a potent tumor-protection mechanism. Indeed, telomerase is not expressed in most human somatic cells, while it is expressed in most cancer cell types (except in a minority of tumors in which telomeres are stabilized through alternative mechanisms) $(1,2)$. Constitutive expression of telomerase in primary, telomerase-negative cells induces immortalization or cooperates with specific oncogenes to induce transformation (3-5).

Cellular senescence, however, might also contribute to a decline in tissue homeostasis by exhausting the supply of progenitors or stem cells, which suggests that organism aging is the trade-off of the evolved adaptation to tumor suppression (antagonistic pleiotropy). As expected from their high replicative potential, stem

\footnotetext{
Address correspondence to: Pier Giuseppe Pelicci, Department of Experimental Oncology, European Institute of Oncology, Via Ripamonti 435, 20141 Milan, Italy. Phone: 39-2-57489831; Fax: 39-2-57489851; E-mail: pgpelicci@ieo.it.

Conflict of interest: The author has declared that no conflict of interest exists.
}

cells express telomerase. They are not, however, immortal and undergo telomere erosion during aging. Notably, overexpression of telomerase in mouse hematopoietic stem cells prevents telomere erosion yet has no effect on the cells' lifespan suggesting that telomere-independent mechanisms regulate replicative senescence of stem cells in vivo $(6,7)$.

Telomerase-deficient mice show progressive telomere shortening. However, they have no obvious defects for the first few generations, most likely because of the unusually long telomeres in the germ line of laboratory mouse strains. After four to six generations, these mutant mice show reduced lifespan, though they lack the full spectrum of classical symptoms of aging. They demonstrate reduced tumor development but, surprisingly, enhanced tumor initiation. This dual effect of telomere shortening has been interpreted to be the consequence of activation of an antiproliferative checkpoint response and induction of chromosomal instability, due to inappropriate fusions of the uncapped telomeres (8-10).

Recent findings shed new light on the molecular pathways associated with the execution of the cellular senescence program and suggest that the tumor-suppressive mechanisms involved may directly contribute to organismal aging, possibly acting at the level of stem/progenitor cells. These topics are addressed by the four review articles (11-14) of this JCI Perspective series.

\section{Both telomere dysfunction and other forms of DNA damage activate $p 53$ and induce senescence} The signal transduction pathways that activate cellular senescence are now better understood. Division of telomerase-negative cells causes the erosion of the telomeric single-stranded 3 '-extension, preventing extension of the double-stranded region and, perhaps more importantly, causing functional uncapping of 
the telomeres. The chromosomal DNA ends are then exposed and recognized by the cell as a double-stranded break. As expected when a double-stranded break forms, DNA-repair and-damage checkpoint factors are recruited to the site of damaged DNA, and a p53-dependent checkpoint is initiated. So replicative senescence is a p53-dependent checkpoint response to DNA damage $(11,12)$.

p53 is a tumor suppressor that is activated by a variety of stressful cellular conditions, including DNA damage, oxidative stress, and oncogenic (hyperproliferative) signals. It is not surprising, then, that the senescence program can be activated by the same stresses, even in telomerase-positive cells - a phenomenon known as premature, or stress-induced, senescence. It is also well recognized that in vitro culturing can be, by itself, a powerful stress (culture shock) that induces premature senescence. For example, Schwann cells and oligodendrocyte precursor cells (which express telomerase) can either grow indefinitely or undergo senescence, depending on culture conditions $(15,16)$. Similarly, human epithelial cells expressing telomerase become immortal only if cultured on feeder layers (17). Therefore, replicative senescence and stress-induced senescence cannot be separated, as they "merely reflect the spectrum of different stimuli that feed into one response program" (11). Moreover, recent findings demonstrate that the rate of telomere shortening of growing cells is influenced by the culture conditions, suggesting that telomere shortening is not simply an intrinsic mitotic counter but is itself regulated by stress signals. In general, it is as if every cell possessed a memory of its history, in terms of both cell divisions and the type of environment to which it was exposed. It is not surprising, then, that damaged DNA is the senescence-activating signal upon telomere shortening. Remarkably, it has been suggested recently that DNA damage is also the signal that limits proliferation of primary cells cultured under standard conditions (18). DNA, indeed, is the only cellular molecule where historical information can be permanently stored, in the form of mutations.

\section{In the absence of $\mathrm{p} 16-\mathrm{Rb}$ or $\mathrm{p} 53$, telomere dysfunction induces genomic instability}

Telomerase and the p53 pathways are not the sole genetic determinants of cellular senescence. The $\mathrm{p} 16-\mathrm{Rb}$ pathway is also involved. Expression of p16 increases during replicative or oncogene-induced senescence and, when increased experimentally, induces premature senescence. Heterochromatin foci are not formed in quiescent cells but are formed in senescent cells, where promoter-bound, hypophosphorylated $\mathrm{Rb}$ permanently represses transcription of a number of cell cycle genes (E2F-target genes) (13). Though this is not definitively ascertained, the p16-Rb pathway can also be activated by telomere dysfunction (11-14). In conclusion, telomere dysfunction, or other DNA damage, activates the senescence program through the p53 and p16-Rb checkpoint pathways.
Based on this simple model, it is not surprising that in vitro, in the absence of functional p53 or p16-Rb which is characteristic of most if not all tumors - telomere dysfunction causes genomic instability and cells accumulate complex chromosome rearrangements. Something similar may also occur during colon and breast tumor development in vivo, where, early in cancer progression, low telomerase activity (and presumably telomere dysfunction) correlates with the presence of complex chromosomal rearrangements (19-21). Subsequently, high levels of telomerase expression are selected to ensure that the cancer cell maintains proper telomere function and growth potential, and the continuous telomerase expression that is needed for tumor growth.

Telomerase, however, may also contribute to tumorigenesis via mechanisms that are independent of its telomere-lengthening effect. Enhanced telomerase expression in telomerase-positive cells promotes growth and survival. When telomerase is overexpressed in mice with telomeres of normal length, it increases the incidence of spontaneous tumors (22). The frequency of mutagen-induced tumors is reduced in early-generation telomere-deficient mice, when telomeres are still long (23). Furthermore, telomerase is periodically expressed in normal human fibroblasts (previously considered telomere-negative), where it participates in the active maintenance of the length of the single-stranded 3 -extension and regulates each cell division (24).

\section{Stem cells are frequent targets of transformation}

How is telomerase expression reactivated in cancer cells, and how are high levels of telomerase expression selected during transformation? Despite intensive investigations, little is known about the physiological mechanisms that regulate telomerase expression. Notably, none of the genes that are most frequently altered in cancer are known to affect telomerase expression (with the possible exception of the myc oncogene). A simple answer to these questions is that the target cell for the initial transforming mutations is telomerase-positive. For most cancers, the target cell of transformation is unknown. Telomerase is expressed in a restricted subset of normal cells: germ cells, stem/progenitor cells, and proliferating lymphocytes. Stem/progenitor cells have a long lifespan, and the machinery for self-renewal is already activated. Therefore, as compared with differentiated cells, they have greater risks of accumulating mutations and may require fewer events to sustain uncontrolled growth. For leukemias, convincing genetic and biological data support the notion that stem/progenitor cells are the most frequent cellular target of transformation (25). Furthermore, it has been recently demonstrated that different types of malignant tumors (breast cancers, glioblastomas, neuroblastomas, and leukemias) contain rare cells with indefinite proliferation potential that support the formation and growth of the remaining tumor population (26). These cells possess the same functional and phenotypic features as the corresponding tissue stem cells and have therefore 


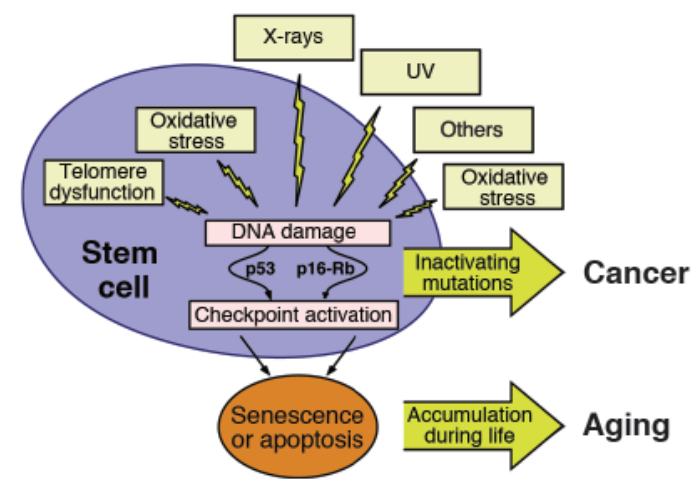

Figure 1

DNA damage accumulates as the consequence of endogenous (telomere dysfunction, oxidative stress) or exogenous (oxidative stress, $\gamma$-irradiation, UV light, and others) attacks. Damaged DNA activates checkpoint responses that are mediated by the p53 and p16-Rb pathways and that result in apoptosis or cellular senescence. If these events occur in stem/progenitor cells, tissue homeostasis is altered - a phenomenon that might contribute to aging. If, instead, DNA mutations that inactivate these checkpoint pathways accumulate, then cancer can arise.

been named tumor stem cells. According to these findings, a tumor can be seen as an abnormal tissue that is initiated by a single, transformed stem cell that retains parts of its program of differentiation.

\section{Inhibition of the senescence program in stem cells contributes to cancer formation}

Regardless of how the problem of telomere lengthening is solved in cancer cells - transformation of telomerasepositive cells or reactivation of telomerase expression in telomere-negative cells - the senescence program has to be suppressed for a cell to evade tumor suppression. In fact, environmental and hyperproliferative stresses are also potent inducers of senescence (and apoptosis) in telomerase-positive cells. The two pathways that execute the program of stress-induced senescence are, as mentioned previously, p19-p53 and p16-Rb, which are invariably altered in cancer (Figure 1). However, since activation of the same two pathways can also trigger apoptosis, it has been difficult to assess the relative contribution of senescence as a tumor-suppressive barrier. Recent findings regarding the Bmi-1 oncogene might help elucidate this issue.

Bmi-1 is a transcriptional repressor that functions as an inhibitor of the senescence program in cultured fibroblasts: its expression is downregulated during spontaneous senescence, and Bmi-1-null fibroblasts enter premature senescence, while enforced Bmi-1 expression extends the replicative lifespan. Bmi-1 regulates senescence through the $\mathrm{p} 16-\mathrm{Rb}$ pathway, by repressing p16 expression. More recently, it has been demonstrated that Bmi-1 functions in vivo to maintain the pool of neuronal and hematopoietic stem cells, by inhibiting their senescence program, again through $\mathrm{p} 16$, while it has no effect on growth and differentiation of precursors $(27,28)$. Bmi-1 might, therefore, con- tribute to tumorigenesis by inhibiting p16-mediated senescence, specifically in stem cells. Notably, Bmi- 1 is frequently amplified in mantle cell lymphomas, and its expression is indispensable for the maintenance of leukemic stem cells (13).

\section{Succesful activation of DNA-damage checkpoint pathways in stem cells (telomere dysfunction, p53) might lead to organism aging}

If inactivation of the senescence program is indeed a relevant mechanism of tumor formation, then its reactivation might represent a successful antitumor strategy. Treatment with various chemotherapeutic agents, such as doxorubicin or other DNA-damage agents, was shown to induce cellular senescence of cancer cells in vitro and, more recently, in vivo $(29,30)$. This event seems to require functional $\mathrm{p} 53$ and $\mathrm{p} 16$. However, at higher doses, doxorubicin induces senescence of p53null cancer cells. Other compounds are now being tested that induce senescence in p53- or p16-null cells, possibly through activation of downstream effectors of these pathways. Reactivation of the senescence program might turn out to be a relevant anticancer strategy, particularly in those tumors where the apoptotic pathways are severely compromised (14).

What happens if, instead, the telomere and the p19-p53 and p16-Rb checkpoints are properly activated in normal stem/progenitor cells? Cells will enter senescence (or apoptosis). If this occurs constantly over the lifespan of an organism, one would intuitively expect a reduction of the pool of stem/progenitor cells, altered tissue homeostasis, and compromised tissue repair, features which may well contribute to organism aging. Remarkably, recent findings suggest that some components of these checkpoint pathways are indeed genetic determinants of lifespan in mammals. I have already commented on the effects of telomerase mutations in mice; in humans, equivalent mutations cause a rare progeroid syndrome. Three different types of transgenic mice have been recently reported, in which p53 expression or activity is to some extent higher than normal (31-33). As expected, all three mouse lines had a markedly lower incidence of cancer. Despite the reduced incidence, these mice did not live longer, and, strikingly, in two of the three lines, lifespan was shorter and the animals showed signs of premature aging. In another model system, genetic intercrosses showed a marked progeric effect of the ATM mutation (ATM is another critical player in cell cycle checkpoints and the regulation of DNA damage) on the telomerase-null genotype (34). Notably, the progeric ATM-telomerase double-mutant mice showed increased p53 levels and apoptosis in various stem cell compartments. Consistently, loss of p53 or p16-p19 increases the pool of stem cells. It appears, in conclusion, that some mammalian tumor-suppressive mechanisms might contribute to aging (Figure 1).

If some tumor-suppressor genes show antagonistic pleiotropy and contribute to aging, then it would be 
impossible to improve tumor-suppressive mechanisms without accelerating aging, and to retard aging without accelerating tumor formation. The few available mutant mice with increased lifespan, however, have no increased risk of cancer. In the case of mice that lack the 66-kDa Shc isoform ( $p 66 \mathrm{Shc}^{-1-}$ mice), available data suggest the existence of a defect in a selective branching of the p53 checkpoint pathway $(35,36)$. p66Shc is a downstream target of p53 and regulates the generation of oxygen radicals, the major source of intracellular oxidative stress. Genetic evidence indicates that the p53-p66Shc signaling pathway is specifically involved in the propagation of proapoptotic oxidative signals (p66Shc). Other functions of p53 are not influenced by p66Shc expression. It appears, therefore, that the tumor-suppressive and aging effects of p53 are mediated by different pathways. If aging is not the cost of tumor suppression, we are then left with one major question: Why do we age? It may be that we are yet too young to provide an answer.

\section{Acknowledgments}

I would like to thank G.F. Draetta, K. Helin, B. Amati, C. Basilico, and J.C. Marine for many helpful and exciting discussions.

1. Kim, N.W., et al. 1994. Specific association of human telomerase activity with immortal cells and cancer. Science. 266:2011-2015.

2. Bryan, T. M., Englezou, A., Dalla-Pozza, L., Dunham, M.A., and Reddel, R.R. 1997. Evidence for an alternative mechanism for maintaining telomere length in human tumors and tumor-derived cell lines. Nat. Med. 3:1271-1274.

3. Bodnar, A.G., et al. 1998. Extension of life-span by introduction of telomerase into normal human cells. Science. 279:349-352.

4. Vaziri, H., and Benchimol, S. 1998. Reconstitution of telomerase activity in normal human cells leads to elongation of telomeres and extended replicative life span. Curr. Biol. 8:279-282.

5. Hahn, W.C., et al. 1999. Creation of human tumor cells with defined genetic elements. Nature. 400:464-468.

6. Yui, J., Chiu, C.P., and Lansdorp, P.M. 1998. Telomerase activity in candidate stem cells from fetal liver and adult bone marrow. Blood. 91:3255-3262.

7. Allsopp, R.C., and Weissman, I.L. 2002. Replicative senescence of hematopoietic stem cells during serial transplantation: does telomere shortening play a role? Oncogene. 21:3270-3273.

8. Rudolph, K.L., et al. 1999. Longevity, stress response, and cancer in aging telomerase-deficient mice. Cell. 96:701-712.

9. Hande, M.P., Samper, E., Lansdorp, P., and Blasco, M.A. 1999. Telomere length dynamics and chromosomal instability in cells derived from telomerase null mice. J. Cell Biol. 144:589-601.

10. Niida, H., et al. 1998. Severe growth defect in mouse cells lacking the telomerase RNA component. Nat. Genet. 19:203-206.

11. Ben-Porath, I., and Weinberg, R.A. 2004. When cells get stressed: an integrative view of cellular senescence. J. Clin. Invest. 113:8-13. doi:10.1172/JCI200420663.
12. Sharpless, N.E., and DePinho, R.A. 2004. Telomeres, stem cells, senescence, and cancer. J. Clin. Invest. In press.

13. Park, I.-K., Morrison, S.J., and Clarke, M.F. 2004. Bmi-1, stem cells, and senescence regulation. J. Clin. Invest. In press.

14. Kahlem, P., Dörken, B., and Schmitt, C.A. 2004. Cellular senescence in cancer treatment: friend or foe? J. Clin. Invest. In press.

15. Mathon, N.F., Malcolm, D.S., Harrisingh, M.C., Cheng, L., and Lloyd, A.C. 2001. Lack of replicative senescence in normal rodent glia. Science. 291:872-875.

16. Tang, D.G., Tokumoto, Y.M., Apperly, J.A., Lloyd, A.C. and Raff, M.C. 2001. Lack of replicative senescence in cultured rat oligodendrocyte precursor cells. Science. 291:868-871.

17. Ramirez, R.D., et al. 2001. Putative telomere-independent mechanisms of replicative aging reflect inadequate growth conditions. Genes Dev. 15:398-403.

18. Parrinello, S., et al. 2003. Oxygen sensitivity severely limits the replicative lifespan of murine fibroblasts. Nat. Cell Biol. 5:741-747.

19. Chin, L., et al. 1999. p53 deficiency rescues the adverse effects of telomere loss and cooperates with telomere dysfunction to accelerate carcinogenesis. Cell. 97:527-538.

20. Artandi, S.E., et al. 2002. Telomere dysfunction promotes non-reciprocal translocations and epithelial cancers in mice. Nature. 406:641-645.

21. Buerger, H., et al. 1999. Comparative genomic hybridization of ductal carcinoma in situ of the breast-evidence of multiple genetic pathways. J. Pathol. 187:396-402.

22. Gonzalez-Suarez, E., et al. 2001. Increased epidermal tumors and increased skin wound healing in transgenic mice overexpressing the catalytic subunit of telomerase, mTERT, in basal keratinocytes. EMBO J. 20:2619-2630.

23. Gonzalez-Suarez, E., Samper, E., Flores, J.M., and Blasco, M.A. 2000. Telomerase-deficient mice with short telomeres are resistant to skin tumorigenesis. Nat. Genet. 26:114-117.

24. Masutomi, K., et al. 2003. Telomerase maintains telomere structure in normal human cells. Cell. 114:241-253.

25. Passegue, E., Jamieson, C.H., Ailles, L.E., and Weissman, I.L. 2003. Normal and leukemic hematopoiesis: are leukemias a stem cell disorder or a reacquisition of stem cell characteristics? Proc. Natl. Acad. Sci. U. S. A. 100(Suppl 1):11842-11849.

26. Al-Hajj, M., Wicha, M.S., Benito-Hernandez, A., Morrison, S.J., and Clarke, M.F. 2003. Prospective identification of tumorigenic breast cancer cells. Proc. Natl. Acad. Sci. U. S. A. 100:3983-3988.

27. Park, I.K., et al. 2003. Bmi-1 is required for maintenance of adult selfrenewing haematopoietic stem cells. Nature. 423:302-305.

28. Molofsky, A.V., et al. 2003. Bmi-1 dependence distinguishes neural stem cell self-renewal from progenitor proliferation. Nature. 425:962-967.

29. Schmitt, C.A., et al. 2002. A senescence program controlled by p53 and p16INK4a contributes to the outcome of cancer therapy. Cell. 109:335-346.

30. te Poele, R.H., Okorokov, A.L., Jardine, L., Cummings, J., and Joel, S.P. 2002. DNA damage is able to induce senescence in tumor cells in vitro and in vivo. Cancer Res. 62:1876-1883.

31. Tyner, S.D., et al. 2002. p53 mutant mice that display early ageing-associated phenotypes. Nature. 415:45-53.

32. Garcia-Cao, I., et al. 2002. "Super p53" mice exhibit enhanced DNA damage response, are tumor resistant and age normally. EMBO J. 21:6225-6235.

33. Davenport, R.J. 2002. Tumor-free, but not in the clear. Sci. Aging Knowl. Environ. 40:nw139.

34. Wong, K.K., et al. 2003. Telomere dysfunction and Atm deficiency compromises organ homeostasis and accelerates ageing. Nature. 421:643-648

35. Migliaccio, E., et al. 1999. The p66shc adaptor protein controls oxidative stress response and life span in mammals. Nature. 402:309-313.

36. Trinei, M., et al. 2002. A p53-p66Shc signalling pathway controls intracellular redox status, levels of oxidation-damaged DNA and oxidative stressinduced apoptosis. Oncogene. 21:3872-3878. 